\title{
Zeeman Relaxation of CaF in Low-Temperature Collisions with Helium
}

\section{Citation}

Maussang, Kenneth, Dima Egorov, Joel S. Helton, Scott V. Nguyen, and John M. Doyle. 2005. Zeeman Relaxation of CaF in Low-Temperature Collisions with Helium. Physical Review Letters 94, no. 12. doi:10.1103/physrevlett.94.123002.

\section{Published Version}

doi:10.1103/physrevlett.94.123002

\section{Permanent link}

http://nrs.harvard.edu/urn-3:HUL.InstRepos:28446851

\section{Terms of Use}

This article was downloaded from Harvard University's DASH repository, and is made available under the terms and conditions applicable to Other Posted Material, as set forth at http:// nrs.harvard.edu/urn-3:HUL.InstRepos:dash.current.terms-of-use\#LAA

\section{Share Your Story}

The Harvard community has made this article openly available.

Please share how this access benefits you. Submit a story.

\section{Accessibility}




\title{
Zeeman relaxation of $\mathrm{CaF}$ in low-temperature collisions with helium
}

\author{
Kenneth Maussang, ${ }^{1,3}$ Dima Egorov, ${ }^{1,3}$ Joel S. Helton, ${ }^{2,3}$ Scott V. Nguyen, ${ }^{1,3}$ and John M. Doyle ${ }^{1,3}$ \\ ${ }^{1}$ Department of Physics, Harvard University, Cambridge, Massachusetts 02138 \\ ${ }^{2}$ Department of Physics, MIT,Cambridge, Massachusetts 02139 \\ ${ }^{3}$ Harvard-MIT Center for Ultracold Atoms, Cambridge, Massachusetts 02138
}

(Dated: February 9, 2005)

\begin{abstract}
The collision-induced Zeeman relaxation rate for collisions of $\mathrm{CaF} X^{2} \Sigma(v \prime \prime=0)$ with ${ }^{3} \mathrm{He}$ is measured to be $\Gamma_{Z}=(7.7+5.4 /-2.5) \times 10^{-15} \mathrm{~cm}^{3} / \mathrm{s}$ at $2 \mathrm{~K}$. This rate is a direct measurement of the influence of spin-rotation coupling on Zeeman relaxation in the first rotational level of CaF. The relationship of this rate to known molecular constants is consistent with recent theory of cold molecular collisions and outlines the ${ }^{2} \Sigma$ molecules conducive to magnetic trapping.
\end{abstract}

PACS numbers: $33.80 . P s, 34.50 .-\mathrm{s}, 34.10 .+\mathrm{x}$

The production of cold polar molecules promises to impact several areas of physics and chemistry. The large electric dipole moments of polar molecules produce a strong interaction that can be exploited for quantum computing [1] or as a source for direct BCS pairing in quantum degenerate molecular matter [2-5]. Also, precision spectroscopy of heavy dipolar molecules in searches for an elementary particle electric dipole moment (EDM) holds tremendous promise for observing physics beyond the Standard Model [6-8]. In the ultra-cold regime, even the smallest activation energy will exceed the available thermal energy. Tunneling becomes the dominant reaction pathway, leading the the exciting possibility of controlling chemical reactions $[9,10]$. Considerable progress has been made in the direct cooling of molecules, yielding larger numbers and colder temperatures in the millikelvin range [11-13]. In addition, photoassociation of bi-alkali mixtures has produced molecules already in the ultracold regime, though vibrationally hot $[14,15]$. Ideally there would exist a ready source of cold molecules of any desired molecular compound that is cold and dense. To achieve this goal, a better understanding of the physics of cold molecular collisions is vital.

While a considerable amount of theoretical work has been done on cold molecular collisions, due to the technical challenges of producing samples of cold molecules, there exists scant experimental data on this subject, despite its central role in the growing field of cold polar molecules [16]. At low temperatures, the collision dynamics are sensitive to the long-range attractive part of the interaction potential, requiring, a quantum mechanical treatment that takes into account all degrees of freedom and the couplings between them. Measurements of low temperature collision rates thus provide a benchmark for quantum mechanical theories of molecular collisions. In particular, low-temperature inelastic molecular collisions are of interest because of their role in cooling and trapping of molecules. In order to magnetically trap molecules, they must be stable against reorientation of their magnetic moments in collisions with other trapped molecules or sympathetic atomic coolant. The reorien- tation of polar molecules, called Zeeman relaxation, is of critical importance as it determines the possibilities for evaporative cooling in a magnetic trap - key to the production of quantum degenerate polar matter.

Buffer-gas cooling and trapping [17] is one technique that has been shown to cool molecules and load them into a magnetic trap[18]. The cooling process relies solely on elastic collisions between molecules and cold helium gas, and so does not require the molecules to have a particular energy level structure or atomic composition. In principle, buffer-gas trapping is applicable to any paramagnetic molecule with a Zeeman sublevel whose energy increases with increasing magnetic field. Such a level is referred to as a low-field seeking state (LFS), whereas a sublevel with decreasing energy is referred to as a highfield seeking state (HFS). However, while collisions with the buffer gas drive translational thermalization and subsequent trapping, they may also induce transitions from the low-field seeking state into the lower energy highfield seeking state. As only molecules in the low-field seeking states are trapped, collision-induced Zeeman relaxation leads to trap loss. In current implementations of buffer-gas trapping, the combined processes of translational thermalization and thermal isolation requires a ratio of elastic to inelastic collisions of $\sigma_{\mathrm{el}} / \sigma_{\mathrm{in}} \geq 10^{4}$ [19]. The Zeeman relaxation rate is therefore a critical parameter in deciding whether a given molecule is suitable for trapping. This rate also indicates how effective evaporative cooling will be in a trapped molecular gas.

Data on Zeeman relaxation rates in cold molecular collisions has thus far been limited to a single measurement in $\mathrm{CaH}\left(X^{2} \Sigma^{+}\right)$-He collisions at $0.4 \mathrm{~K}[18,20]$. Because of the difficulty in calculating detailed molecular potentials, theoretical predictions have been made for only a limited number of molecules [21-23]. These calculations indicate that Zeeman relaxation in ${ }^{2} \Sigma$ molecules occurs via the spin-rotation interaction $\gamma_{\mathrm{sr}} \mathbf{N} \cdot \mathbf{S}$, where $\gamma_{\mathrm{sr}}$ is the spinrotation coupling constant, $\mathbf{N}$ is the rotational angular momentum, and $\mathbf{S}$ is the electronic spin. Zeeman transitions can occur directly through the excited rotational levels but vanish for the ground rotational level $(N=0)$. 


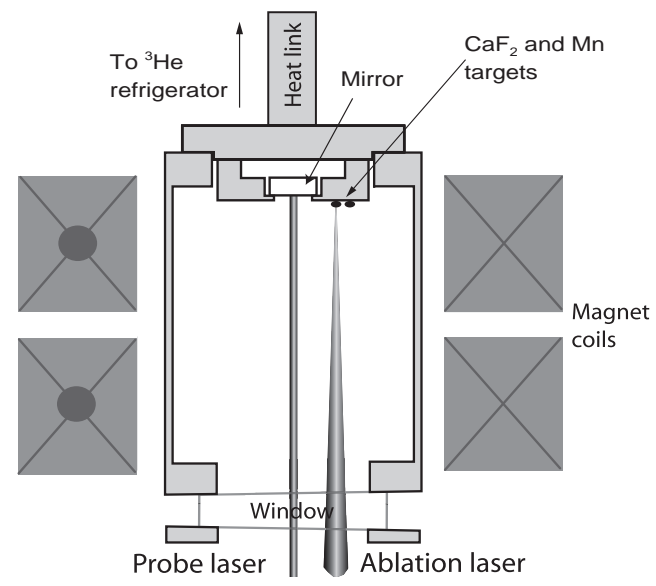

FIG. 1: Schematic of experimental apparatus. The cell is filled with $\sim 10^{16} \mathrm{~cm}^{-3}$ of ${ }^{3} \mathrm{He}$ buffer gas.

In Ref. [24, 25], Krems et al. suggests that Zeeman relaxation in the ground state can still occur through ${ }^{3} \mathrm{He}$ induced electrostatic couplings between the $N=0$ and $N=1$ rotational levels, predicting that the Zeeman relaxation cross-section scales as $\gamma_{\mathrm{sr}}^{2} / B^{4}$, where $B$ is the rotational constant. In this Letter we present a measurement of the Zeeman relaxation rate of $\mathrm{CaF}\left(X^{2} \Sigma^{+}\right)-\mathrm{He}$ collisions due to both the $N=0$ and $N=1$ inelastic channels, compare this measurement with molecular theory, and discuss the possibility of magnetically trapping $\mathrm{CaF}$ and other ${ }^{2} \Sigma$ molecules.

The apparatus, shown in Fig. 1, is described in detail in Ref. [26]. CaF molecules are produced via laser ablation of calcium fluoride $\mathrm{CaF}_{2}$. A cylindrical cell containing a hot-pressed powder pellet of $\mathrm{CaF}_{2}$ is maintained at $0.5 \mathrm{~K}$ by a ${ }^{3} \mathrm{He}$ refrigerator. The cell is filled with ${ }^{3} \mathrm{He}$ gas at a typical density of $10^{16} \mathrm{~cm}^{-3}$. ${ }^{3} \mathrm{He}$ density is monitored by measuring the diffusion time of laser-ablated Mn atoms through the cell [27]. An ablation beam from a pulsed frequency-doubled Nd:YAG laser with a typical pulse energy of $9 \mathrm{~mJ}$ is focused onto the $\mathrm{CaF}_{2}$ sample. Following the ablation pulse, CaF molecules enter the helium gas, thermalize with it translationally and rotationally, and are then lost by diffusion to the cell wall with a typical $1 / e$ time of $\sim 30 \mathrm{~ms}$. Up to $5 \times 10^{13}$ ground-state $\mathrm{CaF}$ molecules are produced per ablation pulse.

The cell is placed coaxially inside the bore of a superconducting solenoid magnet which produces magnetic fields up to $4 \mathrm{~T}$. The external field splits the energies of the Zeeman sublevels $X^{2} \Sigma^{+}(v=0, N=0, M=+1 / 2)$ and $X^{2} \Sigma^{+}(v=0, N=0, M=-1 / 2)$ by $\sim 1.3 \mathrm{~K} / \mathrm{T}$. Immediately following the ablation pulse, the two sublevels are equally populated since the $\mathrm{CaF}$ temperature during ablation is much higher than the Zeeman energy splitting. Collisions with ${ }^{3} \mathrm{He}$ atoms drive both translational thermalization and Zeeman relaxation transitions that gradually bring the populations to their equilibrium

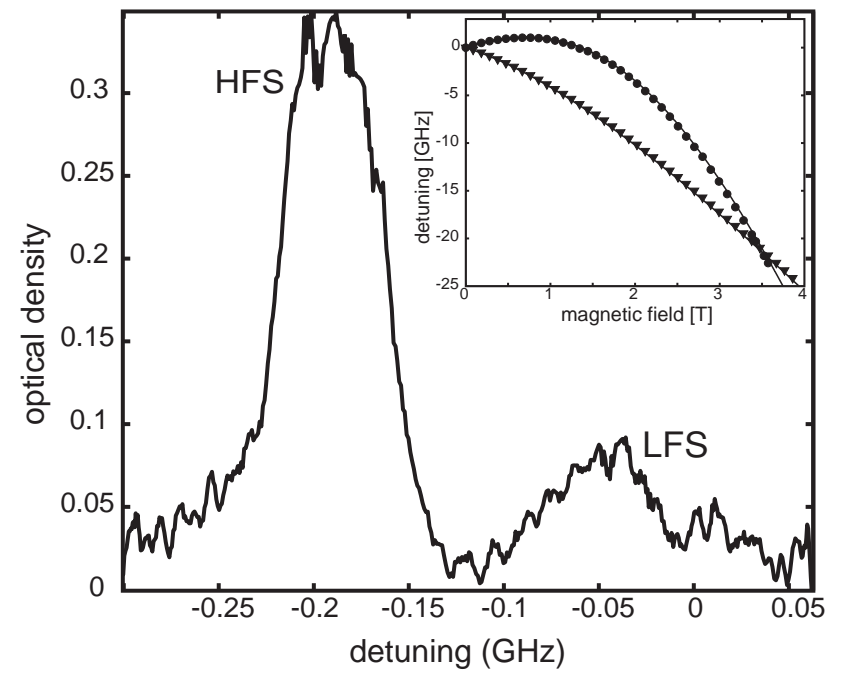

FIG. 2: Spectrum of $\mathrm{CaF}$ at $3.44 \mathrm{~T}$. The peak on the left is from the HFS while the peak on the right is from the LFS. Inset: Zeeman shift of CaF. Circles mark experimental data for LFS and triangles for HFS, while the lines are theoretical predictions (see [28]). Zeeman relaxation measurements were performed near the crossing.

values. By observing the time dependence of the sublevel populations we can determine the Zeeman relaxation rate.

The molecules are detected by laser absorption on the $X^{2} \Sigma^{+}(v=0, J=1 / 2, M=+1 / 2) \rightarrow A^{2} \Pi_{3 / 2}(v=0, J=$ $3 / 2, M=+3 / 2)$ and $X^{2} \Sigma^{+}(v=0, J=1, M=-1 / 2) \rightarrow$ $A^{2} \Pi_{3 / 2}(v=0, N=0, M=-3 / 2)$ transitions at $603 \mathrm{~nm}$. A detection beam from a cavity-stabilized ring dye laser propagates along the centerline of the cell. The beam is linearly polarized along the axis perpendicular to the direction of the magnetic field. Laser frequency, stabilized to sub- $\mathrm{MHz}$ linewidth, is continuously scanned at a rate of $125 \mathrm{~Hz}$ over the absorption line. Scans are fitted to a model spectrum to extract the time dependence of the translational temperature and Zeeman sublevel populations. The relatively high accuracy of this Zeeman relaxation measurement is possible due to the fact that Zeeman shifts of the two transitions cross at $3.55 \mathrm{~T}$ (see Fig. 2 and Ref. [28]). With the magnetic field at $3.44 \mathrm{~T}$, both transitions fall into a single scan of the laser frequency, eliminating a number of measurement uncertainties (Fig. 2).

Fig. 3a shows the time dependence of the measured sublevel population ratio after ablation. It is convenient to define the effective spin temperature $T_{\text {spin }}=$ $-\left(\Delta E_{\mathrm{Z}} / k_{\mathrm{B}}\right) \ln \left[n_{\mathrm{LFS}} / n_{\mathrm{HFS}}\right]$, where $n_{\mathrm{LFS}}, n_{\mathrm{HFS}}$ are populations of low- and high-field seeking states, and $\Delta E_{\mathrm{Z}}$ is the energy splitting between them. The time dependence of the $T_{\text {spin }}$ is shown in Fig. $3 \mathrm{~b}$. The time dependence of the translational temperature is determined by extracting Doppler broadening from the measured spectra. As shown in Fig. 3b, for translational temperatures near the 
equilibrium value, the time dependence is well described by an exponential decay $T_{\mathrm{tr}}(t)=T_{\mathrm{f}}+T_{\mathrm{o}} \exp \left(-t / \tau_{\mathrm{tr}}\right)$, where $T_{\mathrm{f}}$ is the equilibrium translational temperature, $\tau_{\mathrm{tr}}$ is the thermalization timescale, and $T_{\mathrm{o}}$ is a fit parameter. Fig. $3 \mathrm{~b}$ shows the results of the fit; the equilibrium value of the translational temperature was measured to be $T_{\mathrm{f}}=2.0 \pm 0.3 \mathrm{~K}$. This value is higher than the steadystate cell temperature of $0.5 \mathrm{~K}$. We attribute the difference to local heating by the ablation laser. This effect is observed for thermalization of laser ablated Mn atoms as well.

In order to convert the time evolution of the relative Zeeman sublevel populations into a Zeeman relaxation rate, we adopt the following model. The ablation pulse equally populates the Zeeman sublevels. Both populations are lost on equal timescales to diffusion to the walls with a time constant $\tau_{\text {diff }}$. In addition, low field seekers are converted into high field seekers due to Zeeman re- laxation at a rate of $\Gamma_{Z}$, and vice versa due to thermal excitation at a rate of $\Gamma_{\mathrm{Z}} \exp \left(-\Delta E_{\mathrm{Z}} / k_{\mathrm{B}} T_{\mathrm{tr}}\right)$. Because the translational temperature is comparable to the rotational spacing $(n(N=1) / n(N=0) \sim 0.6)$, Zeeman relaxation can occur through both the $N=0$ channel and the $N=1$ channel with rates $\Gamma_{\mathrm{Z}}(N=0)$ and $\Gamma_{\mathrm{Z}}(N=1)$, respectively. Zeeman relaxation through the $N=1$ channel is a three collision process, requiring rotational excitation, Zeeman relaxation, and rotational de-excitation. However, because rotational relaxation rates in other systems are predicted to be quite large $\left(\sim 10^{-10} \mathrm{~cm}^{3} / \mathrm{s}\right)$ [23], we assume here that the rotational levels are always in thermal equilibrium. The three collision process for Zeeman relaxation through the $N=1$ channel can then be approximated as $\Gamma_{\mathrm{Z}}(N=1) \exp \left(-\Delta E_{\mathrm{rot}} / k_{\mathrm{B}} T_{\mathrm{tr}}\right)$, where $E_{\text {rot }}$ is the rotational splitting. The corresponding rate equations for the two Zeeman sublevels are

$$
\begin{aligned}
\frac{\mathrm{d}}{\mathrm{d} t}\left(n_{\mathrm{LFS}}\right) & =n_{\mathrm{He}}\left[\Gamma_{\mathrm{Z}}(N=0)+\Gamma_{\mathrm{Z}}(N=1) \exp \left(\frac{\Delta E_{\mathrm{rot}}}{k_{\mathrm{B}} T_{\mathrm{tr}}(t)}\right)\right]\left[-n_{\mathrm{LFS}}+n_{\mathrm{HFS}} \exp \left(\frac{\Delta E_{\mathrm{Z}}}{k_{\mathrm{B}} T_{\mathrm{tr}}(t)}\right)\right]-\frac{n_{\mathrm{LFS}}}{\tau_{\text {diff }}} \\
\frac{\mathrm{d}}{\mathrm{d} t}\left(n_{\mathrm{HFS}}\right) & =n_{\mathrm{He}}\left[\Gamma_{\mathrm{Z}}(N=0)+\Gamma_{\mathrm{Z}}(N=1) \exp \left(\frac{\Delta E_{\mathrm{rot}}}{k_{\mathrm{B}} T_{\mathrm{tr}}(t)}\right)\right]\left[n_{\mathrm{LFS}}-n_{\mathrm{HFS}} \exp \left(\frac{\Delta E_{\mathrm{Z}}}{k_{\mathrm{B}} T_{\mathrm{tr}}(t)}\right)\right]-\frac{n_{\mathrm{HFS}}}{\tau_{\mathrm{diff}}},
\end{aligned}
$$

where $n_{\mathrm{He}}$ is the ${ }^{3} \mathrm{He}$ density and $T_{\mathrm{tr}}(t)$ is the translational temperature of the $\mathrm{CaF}$ molecules. We define the total Zeeman relaxation rate as the sum of the $N=0$ rate and the $N=1$ rate, $\Gamma_{\mathrm{Z}}=\Gamma_{\mathrm{Z}}(N=0)+\Gamma_{\mathrm{Z}}(N=$ $1) \exp \left(\frac{\Delta E_{\mathrm{rot}}}{k_{\mathrm{B}} T_{\mathrm{tr}}(t)}\right)$. From Eq. 1 and 2 , the population ratio $R=n_{\mathrm{LFS}} / n_{\mathrm{HFS}}$ can easily be shown to follow

$$
\frac{\mathrm{d}}{\mathrm{d} t} R=n_{\mathrm{He}} \Gamma_{\mathrm{Z}}\left[(1+R) \mathrm{e}^{\left(\frac{\Delta E_{\mathrm{Z}}}{k_{\mathrm{B}} T_{\mathrm{tr}}(t)}\right)}-R-R^{2}\right]
$$

with the initial condition $\left.\left(\frac{n_{\mathrm{LFS}}}{n_{\mathrm{HFS}}}\right)\right|_{t=0}=1$ where $t=0$ is the time of the ablation pulse. Eq. 3 can be numerically integrated for given values of $n_{\mathrm{He}}, T_{\mathrm{tr}}(t)$, and $\Gamma_{\mathrm{Z}}$. A leastsquares fit of the measured time evolution of the population ratio with $\Gamma_{Z}$ as the fit parameter yields a Zeeman relaxation rate of $\Gamma_{Z}=(7.7+5.4 /-2.5) \times 10^{-15} \mathrm{~cm}^{3} / \mathrm{s}$, corresponding to a cross-section of $\sigma_{\mathrm{Z}}^{\mathrm{CaF}-\mathrm{He}}=(6.3+$ $4.4 /-2.1) \times 10^{-19} \mathrm{~cm}^{2}$. The dominant source of systematic error is our method for determining $n_{\mathrm{He}}$ which relies on knowledge of the Mn-He diffusion cross-section [27].

By comparing the diffusion time of $\mathrm{CaF}$ and $\mathrm{Mn}$ for a range of $n_{\mathrm{He}}$, we are able to determine the diffusion crosssection for CaF-He collisions to be $\sigma_{\text {diff }}^{\mathrm{CaF}-\mathrm{He}}=(8.1+$ $5.7 /-2.4) \times 10^{-15} \mathrm{~cm}^{2}$. The ratio of elastic-to-inelastic collisions for $\mathrm{CaF}$ then is $\sigma_{\mathrm{diff}}^{\mathrm{CaF}-\mathrm{He}} / \sigma_{\mathrm{Z}}^{\mathrm{CaF}-\mathrm{He}}=(1.3+$ $1.3 /-0.5) \times 10^{4}$.
The measured value for $\Gamma_{Z}^{\mathrm{CaF}-\mathrm{He}}$ is much larger than the previously measured value for the Zeeman relaxation rate of $\mathrm{CaH}$ at $0.4 \mathrm{~K}$ of $\Gamma_{\mathrm{Z}}^{\mathrm{CaH}-\mathrm{He}}=10^{-17 \pm 1} \mathrm{~cm}^{3} / \mathrm{s}[20]$. The molecular constants relevant to Zeeman relaxation for these two molecules are different. $\mathrm{CaH}$ has a large rotational splitting of $B^{\mathrm{CaH}}=4.277 \mathrm{~cm}^{-1}$ compared to $\mathrm{CaF}, B^{\mathrm{CaF}}=0.339 \mathrm{~cm}^{-1}[29]$. At $0.4 \mathrm{~K}$ the Boltzmann factor for the $N=1$ state in $\mathrm{CaH}$ is $\sim 10^{-14}$. Zeeman relaxation in the $\mathrm{CaH}$ measurement must proceed through the $N=0$ channel. As stated previously, at $2 \mathrm{~K}$ Zeeman relaxation in $\mathrm{CaF}$ will occur through both the $N=0$ and $N=1$ channels. Since the ground state channel is a second-order process, $\Gamma_{Z}^{\mathrm{CaF}-\mathrm{He}}$ should be dominated by $\Gamma_{\mathrm{Z}}(N=1)$. We are able to place an upper-bound on the $N=0$ rate of $\Gamma_{\mathrm{Z}}(N=0)<7.7 \times 10^{-15} \mathrm{~cm}^{3} / \mathrm{s}$.

Our measurement does allow for comparison of Zeeman relaxation in the $N=1$ level to the spin-rotation interaction. In the $N=1$ level, the Zeeman relaxation crosssection should scale as $\gamma_{\mathrm{sr}}^{2}[24,25]$. The spin-rotation coupling constant of $\mathrm{CaF}$ is $\gamma_{\mathrm{sr}}^{\mathrm{CaF}}=1.3 \times 10^{-3} \mathrm{~cm}^{-1}$ while that of $\mathrm{CaH}$ is $\gamma_{\mathrm{sr}}^{\mathrm{CaH}}=41.5 \times 10^{-3}$. Krems et al. has calculated a rate of $\Gamma_{\mathrm{Z}}^{\mathrm{CaH}-\mathrm{He}}(N=1)=(2 \pm 5) \times 10^{-12} \mathrm{~cm}^{3} / \mathrm{s}$ for $\mathrm{CaH}$ at $0.4 \mathrm{~K}$ [30]. Using $\gamma_{\mathrm{sr}}$ and adjusting for the translational temperature difference, the scaling dependence predicts that $\Gamma_{\mathrm{Z}}^{\mathrm{CaH}-\mathrm{He}}(N=1)$ is $\sim 500$ times faster than $\Gamma_{\mathrm{Z}}^{\mathrm{CaF}-\mathrm{He}}(N=1)$, falling within the error allowed by both the measurement and theory. 

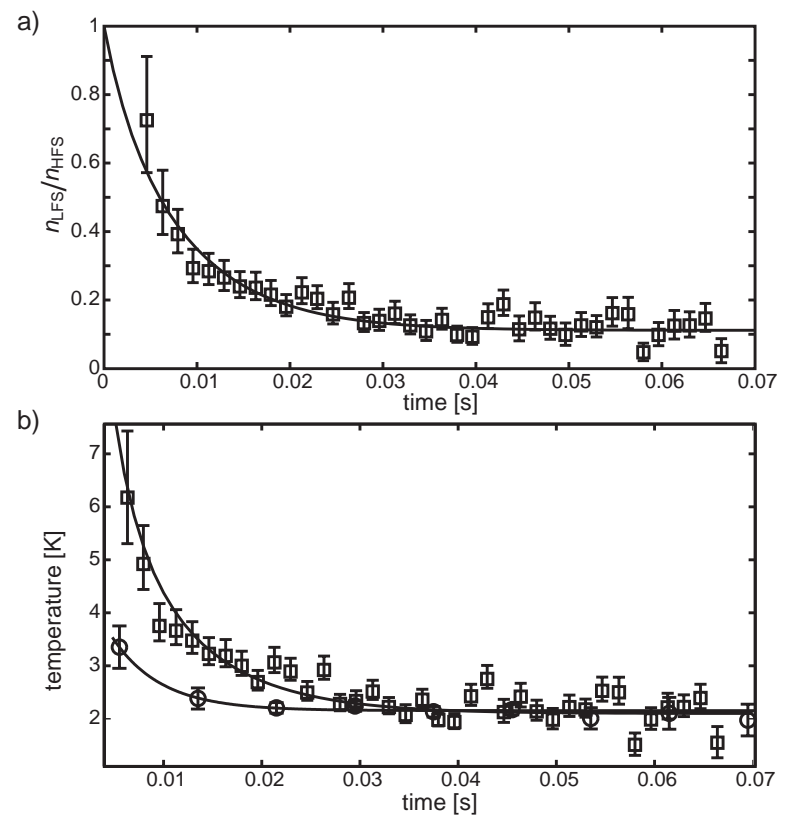

FIG. 3: a) Dependence of the ratio of the Zeeman sublevel populations on time after ablation. Line is a fit to the rate equation solution. b) Translational and spin temperature equilibration. Circles are translational temperatures calculated from measured Doppler broadening. Squares are spin temperatures calculated from measured Zeeman sublevel populations. Lines are fits to theoretical models.

As expected from theory, the measured CaF elasticto-inelastic ratio is approaching the limiting value where current buffer-gas trapping techniques can successfully trap molecules. This ratio can be improved by lowering the ${ }^{3} \mathrm{He}$ buffer gas temperature to suppress the population in the $N=1$ rotational level. In addition, choosing ${ }^{2} \Sigma$ molecules with large rotational constants such as the hydrides would further suppress the populations. Molecules with small spin-rotation constants are another possibility. However, $\gamma_{\mathrm{sr}}$ for $\mathrm{CaF}$ is already relatively small. Typical values for ${ }^{2} \Sigma$ molecules range from $\gamma_{\mathrm{sr}}=0.001-1 \mathrm{~cm}^{-1}$. If the excited state channel can be neglected (e.g. by cooling to $0.4 \mathrm{~K})$, the $\gamma_{\mathrm{sr}}^{2} / B^{4}$ scaling of $\Gamma_{\mathrm{Z}}(N=0)$ predicted by theory points toward molecules with large rotational constants and small spinrotation constants. Finally, new buffer-gas techniques are being developed that have fewer in-field collisions with helium[31]. As such, molecules even with very small rotational constants could be buffer-gas cooled.

In conclusion, we have measured the inelastic relaxation rate of $\mathrm{CaF}$ in collisions with $\mathrm{He}$ and find it to be consistent with theory. This measurement, taken with the previous result for $\mathrm{CaH}$, indicate that molecules with low spin-rotation and/or high rotational constants are amenable to buffer-gas loading. Similarly, we can expect that molecule-molecule collision rates for species with similar molecular constants will be favorable for evaporative cooling. For trapping of other polar molecules, those with high spin-rotation and low rotational constants, these findings underline the importance of using high-field-seeking traps (e.g. FORT [32], Microwave traps [33]), where inelastic two-body collisions can be avoided altogether.

This work was supported by the NSF through the Harvard/MIT Center for Ultracold Atoms and the DOE. We thank B. Friedrich and R. Krems for helpful discussions.

[1] D. DeMille, Phys. Rev. Lett. 88, 067901 (2002)

[2] L. Santos et al.,Phys. Rev. Lett. 85, 1791 (2002)

[3] M. Baranov et al., Phys. Scr. 74, T102 (2002)

[4] L. D. Carr, G. V. Shlyapnikov and Y. Castin, Phys. Rev. Lett. 92, 150404 (2004)

[5] M. A. Baranov, Ł. Dobrek and M. Lewenstein, Phys. Rev. Lett. 92, 250403 (2004)

[6] E.A. Hinds, Physica Scripta T70, 34 (1997)

[7] J.J. Hudson et al., Phys. Rev. Lett. 89023003 (2002)

[8] D. DeMille et al., Art and Symmetry in Experimental Physics: Festschrift for Eugene D. Commins. (AIP Conf. Proc. 596, 2001)

[9] N. Balakrishnan and A. Dalgarno, Chem. Phys. Lett. 341, 652 (2001)

[10] E. Bodo, F.A. Gianturco, and A. Dalgarno, J. Chem. Phys. 116, 9222 (2002)

[11] D. Egorov et al., Euro. Phys. Journal D 31, 307 (2004)

[12] T. Junglen et al., Euro. Phys. Journal D 31, 365 (2004)

[13] van de Meerakker et al., Phys. Rev. Lett. 94, 023004 (2005)

[14] M.W. Mancini et al., Phys. Rev. Lett. 92, 133203 (2004)

[15] A.J. Kerman et al., Phys. Rev. Lett. 92, 153001 (2004)

[16] see, for example, contributions to 2004 ITAMP Workshop on Ultracold Polar Molecules.

[17] R. deCarvalho et al., Eur. Phys. J. D. 7, 289 (1999).

[18] J. D. Weinstein et al., Nature, 395, 148 (1998).

[19] C. I. Hancox et al., Nature. 431, 281 (2004).

[20] J. D. Weinstein, Ph.D thesis, Harvard University, 2002.

[21] R. V. Krems et al., Phys. Rev. A 68, 051401(R) (2003).

[22] J. L. Bohn, Phys. Rev. A 62, 032701 (2000).

[23] N. Balakrishnan et al., J. Chem. Phys. 118, 7386 (2003).

[24] R. V. Krems and A. Dalgarno, J. Chem. Phys.120, 2296 (2004).

[25] R. V. Krems et al., Phys. Rev. A 67, 060703(R) (2003).

[26] J. G. E. Harris et al., Europhys. Lett. 67, 198 (2004).

[27] S. V. Nguyen et al., (unpublished).

[28] R. V. Krems et al., J. Chem. Phys. 121, 11639 (2004)

[29] M. Mizushima, The theory of rotating diatomic molecules. (John Wiley \& Sons, New York, 1975).

[30] R. V. Krems, (unpublished).

[31] S.E. Maxwell et al. (unpublished).

[32] J. D. Miller and R. A. Cline and D. J. Heinzen, Phys. Rev. A 47, R4567 (1993)

[33] D. DeMille, D.R. Glenn, and J. Petricka, Euro. Phys. Journal D 31, 375 (2004) 